\title{
Cálculo da evapotranspiraçāo real na Bacia Amazônica através do método aerológico
}

\author{
José Marques (*) \\ Eneas Salati $(* *)$ \\ Jesus Marden dos Santos $(* * *)$
}

\section{Resumo}

No presente trabalho, são apresentados os dados obtidos para a evapotranspiração real (ER) utilizandose o "método aerológico", o qual se fundamenta no cálculo da divergência do campo do fluxo do vapor d'água na troposfera sobre a região Amazônica. raz-se um estudo comparativo com os demais resultados publicados por outros autores empregando outras metodologias e tenta-se estabelecer os limites para o valor de ER na região. Utilizando-se dados do período 1972/ 1975 , estimou-se em $1260 \mathrm{~mm} /$ ano o valor da ER na região Amazônica.

\section{INTRODUÇÃO}

A evapotranspiração real, ou seja as saídas de água de um ecossistema através da transpiração das plantas e da evaporação do solo, é um dos parâmetros mais difíceis de medir-se no balanço hídrico. Medidas da evapotranspiraçãc real em pequenas culturas têm sido feitas através de lisímetros mas a utilização destes equipamentos é impraticável em ecossistemas naturais, especialmente em florestas. A estimativa da evapotranspiração real em ecostistemas naturais, abrangendo grandes áreas, é feita por métodos indiretos estimando-se primeiramente a evapotranspiração potencial à qual são aplicados coeficientes adequados, (estima-se a evapotranspiração real). Métodos clássicos como os de Blaney \& Criddle (1950), Turc (1955), Thornthwaite (1955), Penman (1956) e Budyko (1956) têm sido utilizados para estas estimativas.

Em bacias hidrográficas bem definidas pode-se também estimar a evapotranspiração real através de medidas da precipitação, do escoa- mento superficial e do escoamento subterrâneo. No caso específico de estimar-se a evapotranspiração potencial e a evapotranspiração real para a bacia Amazônica, o problema tơnnase bem mais complexo, pelas dimensões da região e principalmente pela escassez de dados básicos necessários.

Através de aproximações sucessivas e utilizando métodos distintos, tem sido possível estabelecer as ordens de grandeza dos com. penentes do ciclo hidrológico para a bacia Amazônica. Molion (1975), Villa Nova et al. (1976), Marques $(1976,1978)$, Marques et al. (1977), Dall'Olio (1976); para Manaus Ribeiro (1976) ; para todo o Brasil, DMET (1972) e para grande parte da região do Norte, IPEAN (1972).

No presente trabalho, são apresentados dados obtidos utilizando-se do "método aerológico", o qual se fundamenta no cálculo da divergência do campo do transporte do vapor d'água na troposfera sobre a região Amazônica.

Este método tem sido amplamente utilizado para a estimativa da evaporação em grandes áreas. Assim, Nyberg (1958) e Vaisänen (1962) aplicaram-no à Finlândia; Nyberg (1965), à Suécia; Palmén \& Söderman (1966) ao Mar Báltico, e Alestalo \& Savijärvi (1977) ao Nordeste da Europa.

Faz-se um estudo comparativo com os demais dados existentes e tenta-se estabelecer os limites para o valor da evapotranspiração real na região.

Este trabalho faz parte de um estudo mais amplo da Hidrologia da região denominado "A divergência do campo do transporte de vapor

\footnotetext{
( $\left.{ }^{*}\right)$ - UFRJ - I. Geociêncios, Departamento de Meteorologia.

("*) - ESALQ/USP - Departamento de Física e Meteorologia e Instituto Nacional de Pesquisas da Amazônia, Manaus.

$\left.{ }^{(* *)}\right)$ - CENA - Centro de Energia Nuclear na Agricultura.
} 
d'água na troposfera e a hỉdrologia da bacia Amazônica" cujos demais resultados serão oportunamente divulgados.

\section{MATERIAIS E MÉTODOS}

Os dados de precipitação $(\mathrm{P})$ utilizados no presente estudo foram obtidos a partir do levantamento dos totais mensais em 117 estações na região e adjacências, cobrindo o período 1972/1975. Os dados básicos do ar superior tais como temperatura $(T)$, umidade relativa (UR) e vento (V), foram obtidos a partir da rede de estações de radiossondagem na região e adjacências, para o mesmo período, através da análise de um total de 9348 radiossondagens (figura 1). A partir desses dados foram computados os campos do fluxo de vapor d'água atmosférico $\overrightarrow{(Q)}$ bem como a sua divergência $(\nabla \vec{\nabla})$ na região, cuja metodologia de cálculo se acha detalhada em Marques (1976 e 1978).

Para médias anuais e grandes regiões a equação do balanço pode ser descrita na fórmula abaixo :

$$
\begin{aligned}
& \frac{\partial}{\partial t} \int \frac{1}{g} \bar{q} d p+\nabla \cdot \int \overrightarrow{q V} \overrightarrow{d p}=-\frac{1}{g} \\
& \left(\bar{q}(\dot{w})_{s}+\frac{1}{g}\left(\bar{q}{ }_{c} \dot{\omega}\right)_{s} \ldots \ldots \ldots \ldots(1)\right.
\end{aligned}
$$

onde os termos

$$
\begin{gathered}
\vec{Q}=\frac{1}{g} \int \overline{q V d P}-\begin{array}{l}
\text { representa o campo } \\
\text { de escoamento aéreo } \\
\text { de vapor d'água. }
\end{array} \\
W=\frac{1}{g} \int \overline{q d P}-\begin{array}{l}
\text { representa o armaze- } \\
\text { namento aéreo de va- } \\
\text { por d'água. }
\end{array} \\
E=-\frac{1}{g}(\bar{q}(\omega)) \quad s \quad \text { é o fluxo vertical as- } \\
\text { cendente d e vapor } \\
\text { d'água que atravessa }
\end{gathered}
$$

uma superfície $s$, re presentado pela eva. potranspiração real.

$$
P=\frac{1}{g}\left(\ddot{q}_{c} \dot{\omega}_{c}\right)-\text { é o fluxo vertical }
$$

descendente de áqua na fase condensada $c$ que atravessa uma superfície $s$, representada pela precipitação.

Para a bacia Amazônica, verificou-se que a variação do armazenamento aéreo do vapor d'água é desprezível quando comparado com os demais termos da expressão (1), o que permite utilizá-la na seguinte fórmula simplificada:

$$
E=P+\nabla \cdot \vec{Q} \ldots \ldots \ldots \ldots
$$

A partir dos dados publicados pelo DMET (1972), estimou-se que a evapotranspiração potencial é da ordem de 1452 e a real 1306. Segundo os dados publicados pelo IPEAN (1972) utilizando o método de Thornthwaite, estimouse a evapotranspiração potencial em $1475 \mathrm{~mm} /$ ano e a real em $1320 \mathrm{~mm} /$ ano.

$\mathrm{Na}$ tabela 1, estão resumidos os valores obtidos pelos diversos autores e por métodos independentes. Análises dos dados obtidos até o momento utilizando maior número de informações publicadas levam-nos a concluir que, tomando-se a região Amazônica como um todo, sem levar em consideração os problemas de ecossistemas específicos, o valor da evapotranspiração real deve estar entre os limites de 1146 a 1260 .

\section{RESUltados E DISCUSSÃo}

Molion (1975) utilizando o "método climatonômico" e através de hipóteses aproximadas, estimou que a evapotranspiração real para a bacia Amazônica seria da ordem de $1146 \mathrm{~mm}$ por ano. Nestas condições o balanço hídrico para a bacia Amazônica seria de 2379 para precipitação de 1234 para vazão (escoamento superficiall. 


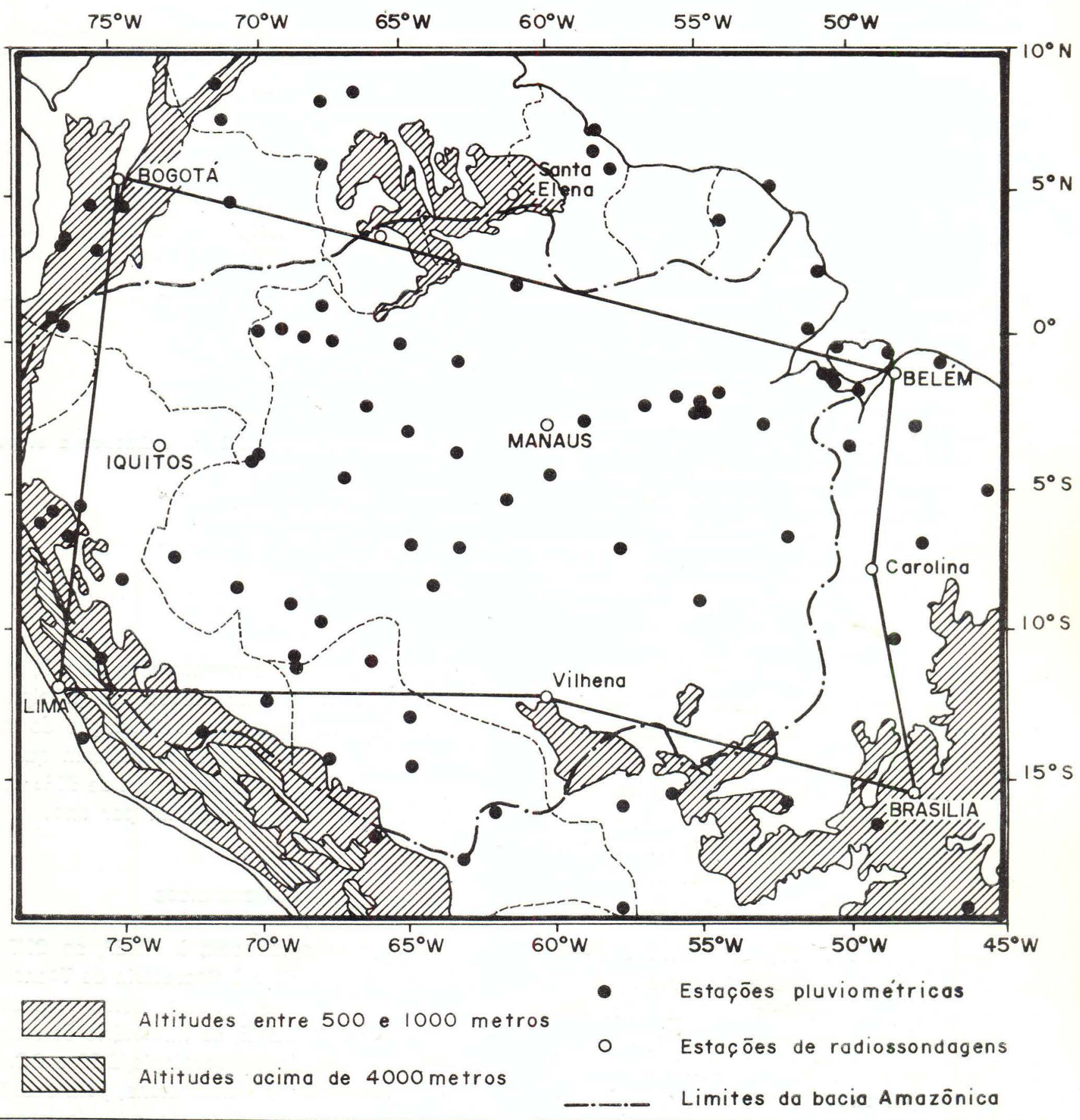

Fig. 1 - Rede de estações pluviométricas e de radiossondagens utilizadas.

Villa Nova et al. (1976) aplicando o método de Pénman adaptado para florestas por Shiau et al. (1973), utilizando dados de 18 postos meteorológicos na Amazônia brasileira estimou a evapotranspiração potencial como sendo, em média, $4 \mathrm{~mm}$ por dia ou seja $1460 \mathrm{~mm}$ por ano. Embora na parte mais ocidental (alto rios $\mathrm{Ne}$ - gro e Solimões) não haja déficit de água no solo, em outras regiões existem períodos de seca bem definidos, devendo-se assim admitir que nestas áreas a evapotranspiração real média anual seja menor do que a evapotranspiração potencial. O fator a ser utilizado para estimar-se a evapotranspiração real é difícil de Cálculo... 
TABELA 1 - Valores encontrados no presente trabalho, comparados com os de outros autores empregando outras metodologias.

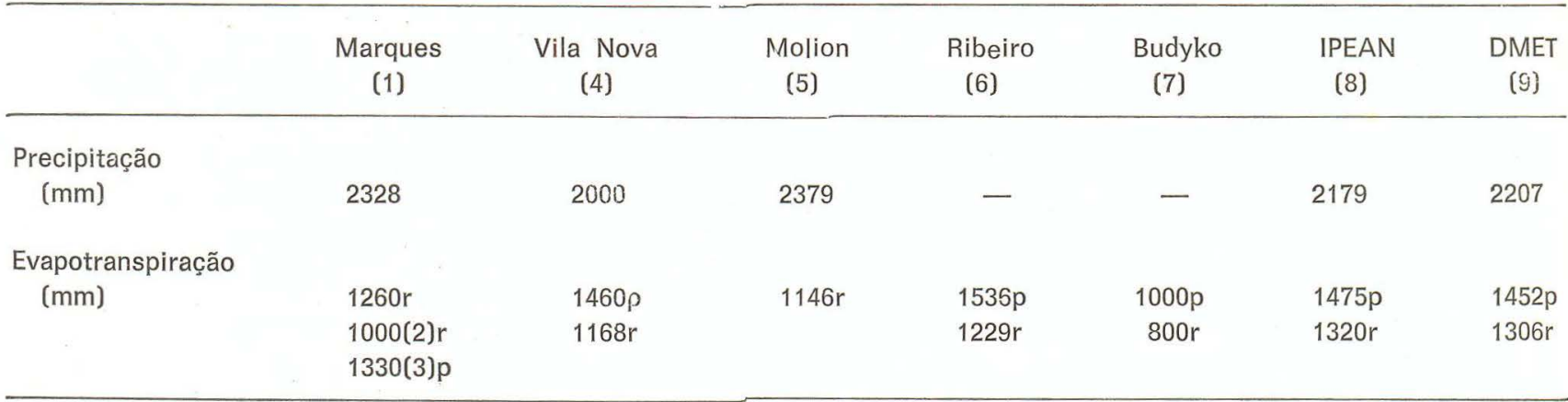

$\mathrm{p}=$ potencial

$r=$ real

(1) Método aerológico; média do período 1972/1975.

(2) Método aerológico; ano 1972, trecho Belém-Manaus.

(3) Método de Thornthwaite; ano 1972, trecho Belém-Manaus.

(4) Método de Penman adaptado para florestas; período 1931/1960 na maioria das estações; estimou-se a evapotranspiração real como sendo $80 \%$ da potencial

(5) Método climatonômico; período 1931/1960.

(6) Método de Thornthwaite; período 1965/1973; Reserva Florestal Ducke, próxima a Manaus.

(7) Citado por Molion (1975), estimou-se a evapotranspiração real como sendo $80 \%$ da potencial.

(8) Método de Thornthwaite; período acima de 10 anos; médias de 7 estações.

(9) Método de Thornthwaite; períodos diversos. Média para as mesmas 7 estações usadas em (8).

ser calculado. Através dos mapas do RADAMBRASIL estimaram-se as áreas cobertas por florestas e, considerando-se os períodos sem falta de água no solo, concluiu-se que um fator da ordem de 0,7 a 0,8 deve ser aplicado ao valor da evapotranspiração potencial para obter-se valor da evapotranspiração real. Com esses dados, foram obtidos o valor de $1168 \mathrm{~mm}$ por ano para a evapotranspiração real.

Marques et al. (1977) estudando a divergência do campo do fluxo de vapor d'água entre Belém e Manaus utilizando dados de radiossondagem de 1972 estimaram a evapotranspiração real nesta região como sendo da ordem de $1000 \mathrm{~mm}$ por ano.

Ribeiro (1976) analisando os dados obtidos durante 9 anos na Reserva Florestal Ducke, nas proximidades de Manaus, estimou a evapotranspiração potencial pelo método de Thornthwaite como sendo igual a $1536 \mathrm{~mm}$.

Dall'Olio (1976) e Salati et al. (1979) evidenciaram através de medidas das concentrações de ${ }^{18} \mathrm{O}$ em águas de chuva, uma forte recirculação do yapor d'água dentro da bacia Amazônica. A fim de interpretar os valores encontrados para as concentrações de ${ }^{18} \mathrm{O}$, adimitiram que em alguns meses das precipitações são provenientes da recirculação do vapor d'água. Os dados obtidos indicam que a evapotranspiração real é da ordem de $50 \%$ das precipitações, ou seja $1200 \mathrm{~mm}$ por ano.

\section{AGRAdECIMENTOS}

Os autores agradecem à FINEP, ao CNPq, à FAPESP, ao CNEN e à Secretaria de Tecnolo. gia do Estado de São Paulo, pelos recursos concedidos; à SUDENE, ao Ministério da Aeronáutica (Divisão de Meteorologia/DEC e DCA/ CTA e à Força Aérea Venezuelana, pela cessão dos dados meteorológicos de altitude necessários a esta pesquisa.

\section{SUMMMARY}

Actual evapotranspiration (E) data obtained by the "aerological method" based on tropospheric water vapor field divergence over Amazonas basin, are presented. Confront was made with evapotranspiration data obtained by other methodologies; E limits were stablished for the region with a mean value of $1260 \mathrm{~mm} /$ year for the 1972 to 1975 period. 


\section{BIBLIOGRAFIA}

Alestalo, M. \& SAviJârvi

1977 - Experiences with the use of the aerological method in evaporation studies in Northwestern Europe. Nordic Hydrology, 8 (1): 47-56.

Blaney, H. F. \& CRIDdle, W.D.

1950 - Determining water requeriments in irrigated areas from climatological and irrigation data. U.S. Dept. Agric., Soil Cons. Service, Tech. Paper 96.

BUDYKO, M.I.

1956 - Heat budget of the earth's surface. Off. Tech. Serv., U.S. Dept. Commerce, Wash. DC

Dall'Olio, A.

1976 - A composição isotópica das precipitações do Brasil: modelos isotérmicos e a influência da evapotranspiração na bacia Amazônica. Tese de Mestrado, ESALQ/USP, 180p.

Departamento Nacional da Produção Mineral

1974/1978 - Projeto RADAMBRASIL, V. 1-18.

Instituto de Pesquisas Agropecuárias do Norte

1972 - Zoneamento Agrícola da Amazônia. Bol. Tec. IPEAN, Belém, 54

DEPARTAMENTO NACional dE METEOROLOGIa

1972 - Balanço hídrico do Brasil. 135p.

MARQUES, J.

1976 - Contribuição ao estudo hidrológico da bacia Amazônica. Tese de Mestrado, ESALQ/USP. $116 p$.

1978 - A transferência horizontal de vapor d'água na troposfera e a hidrologia da Bacia Amazônica. Tese de Doutoramento. Esc. Sup. Agricultura "Luis de Queiroz/USP. 112p.

Marques, J.; Santos, J.M. dos; Villa Nova, N.A \& SALATI, E.

1977 - Precipitable water and water vapor flux between Belém and Manaus. Acta Amazonica, Manaus, 7 (3): 355-362.

Molion, L.C.B.

1975 - A climatonomic study of the energy and moisture fluxes of the Amazonas basin with considerations of deforestation effects. PhD. Thesis, University Wisconsin, USA.
NYBERG, A.

1958 - A determination of some monthly values of evapotranspiration in Finland and in a region in the northastern Atlantic. Geophysica, 6:377-387.

1965 - A comparative of the evaporation in Southern Sweden during 1957. Tellus, 17: 473-483.

PALMÉN, E. \& SöDERMAN, D.

1966 - Computations of the evaporation from the Baltic Sea from the flux of water vapor in the atmosphere. Geophysica, 8 (4): 261-279.

Penman, H.L.

1956 - Evaporation: an introductory survey. Neth. J. Agr. Sci., 4 (1): 9-29.

RIBEIRO, M.N.G.

1976 - Aspectos climatológicos de Manaus. Acta Amazonica, 6 (2): 229-233.

Salati, E.; Dall'olio, A.; Matsui, E. \& Gat, J.R.

1979 - Recycling of water in Amazon Basin an isotopic study. Wat. Res. Research, 15 (5): 1250-1258.

SHIAU, S.V. \& DaVAR, K.S.

1973 - Modified Penman method for potencial evapotranspiração from forest regions. Journal of Hydrology, 18: 349-365.

THORNTHWAITE, C.W.

1955 - The water balance. Publications in climatology, 8 (1), Drexel Inst. of Technology, Centerton

TURC, L.

1955 - Le bilan d'eau des sols: relations entre les precipitation l'evaporation et l'èconcement. Versailles, Inst. Nat. Rech. Agr., Lab. des Sols.

VAISÂNEN, A.

1962 - A computation of the evaporation over Finland during a rainless period based on the divergence of the water vapor flux. Geophysica, 8 (2): 159-164.

Vila Nova, N.A.; Salati, E. \& Matsui, E.

1976 - Estimativa da evapotranspiração na bacia Amazônica. Acta Amazonica, 6 (2): 215-228

(Aceito para publicação em 28/09/79) 